\title{
André Gaillard, le passeur décisif des Cahiers du Sud
}

\author{
Christel Brun-Franc
}

URL : http://journals.openedition.org/lcc/420

DOI : $10.4000 /$ lcc. 420

ISSN : $2430-4247$

Éditeur

Université Aix-Marseille (AMU)

\section{Référence électronique}

Christel Brun-Franc, «André Gaillard, le passeur décisif des Cahiers du Sud », Les chantiers de la création [En ligne], 5 | 2012, mis en ligne le 23 janvier 2015, consulté le 08 avril 2020. URL : http:// journals.openedition.org/lcc/420 ; DOI : https://doi.org/10.4000/lcc.420 


\author{
André Gaillard, le passeur décisif des Cahiers du Sud \\ Christel Brun-Franc \\ Université d'Aix-Marseille \\ christel.brunfrancesfr.fr
}

\begin{abstract}
En 1924 arrive aux Cahiers du Sud, petite revue littéraire marseillaise, un jeune homme mal connu aujourd'hui, le poète André Gaillard. Son entrée au comité de rédaction révolutionne la ligne éditoriale de la revue qui se place alors très rapidement à la pointe de l'avant-garde, publiant un certain nombre d'auteurs surréalistes (orthodoxes ou dissidents) ou encore les écrivains du Grand Jeu. Grâce à cet homme, toujours à l'affût des jeunes poètes et à qui Jean Ballard, le directeur, laisse les pleins pouvoirs, les Cahiers entrent en peu de temps dans l'histoire littéraire française.

Cahiers du Sud, André Gaillard, Jean Ballard, revue littéraire,
\end{abstract} Marseille

\begin{abstract}
Les Cahiers du Sud, a minor magazine from Marseilles in 1924, welcomed a young man who remains quite unknown nowadays, the poet André Gaillard. His arrival to the editorial board was a revolution for the editorial policy of the magazine; which quickly became at the vanguard of its time: publishing quite a number of surrealist writers (orthodox or dissidents), or even the writers of Le Grand Jeu. Thanks to this man, always in the lookout for young poets and to whom Jean Ballard, the managing editor, gave carte blanche; the cahiers progressively became a prominent institution, influencing the history of French literature.
\end{abstract}

«Ce qu'il fallait aux Cahiers du Sud c'était un entraineur, un aimant, une vigie. Nous l'eûmes avec André Gaillard » (Ballard, 1963, 26).

Les Cahiers $d u$ Sud sont une revue littéraire qui parait pendant plus de cinquante ans, de 1914 à la fin de 1966, à Marseille. Créée par Marcel Pagnol et quelques-uns de ses amis du lycée, la revue prend d'abord pour titre Fortunio. En 1925, lorsqu'elle est rebaptisée Les Cahiers $d u$ Sud et que Jean Ballard prend officiellement sa direction, rien ne la prédispose à devenir une grande revue. Pourtant, elle se place rapidement au premier rang de l'actualité littéraire entre 1925 et 1939. Si le travail et la volonté de Jean Ballard sont, à l'évidence, des 
facteurs essentiels de ce succès étonnant, un jeune poète, André Gaillard, va donner à la revue une jeunesse et une impulsion déterminantes. Ballard, en effet, était déjà présent dans le comité de rédaction de Fortunio qui, jusqu'à la fin de 1923, s'intéressait assez peu à l'avantgarde. Même lorsque certaines critiques sur le surréalisme seront ensuite publiées, leurs auteurs se prononcent avec beaucoup de réserves : Gabriel d'Aubarède dans son compte rendu sur A la dérive de Philippe Soupault en mars 1924 regrette, par exemple, de «voir employé à ces jeux stériles des dons incontestables » (321). Si Fortunio apparaît alors comme une revue assez conformiste, il est étonnant de constater que, dès 1923-1924, sont présents dans Le Feu, revue aixoise pourtant provençalisante, des articles enthousiastes sur Eluard, Péret ou Delteil. Et qui collaborait alors au Feu? André Gaillard, bien sûr.

Né en 1898, il se lance dans l'écriture après la rupture provoquée par la Première Guerre mondiale. Ses poèmes paraissent dans plusieurs revues provinciales, dont $\mathrm{Le} \mathrm{Feu,} \mathrm{lorsqu'il} \mathrm{est}$ amené à s'installer à Marseille en 1920 pour raisons professionnelles. Ces œuvres de jeunesse ne sont pas vraiment remarquables ${ }^{1}$ et ne laissent rien présager de son évolution littéraire. Rapidement pourtant, il délaisse les vers élégiaques et ronsardisants, qualifiés souvent de fantaisistes $^{2}$, pour se tourner vers l'avant-garde surréaliste : il a rencontré, lors de plusieurs séjours parisiens, René Crevel, André Breton ou encore Paul Eluard. Ce changement est perceptible dès 1924 dans les textes qu'il publie dans le Feu. Il commence à lire des auvres anticonformistes, celles de Delteil, Morand ou Soupault, ce qui lui permet de renouveler son lyrisme et sa conception de la poésie. Présenté à Ballard en 1924 par Léon-Gabriel Gros ${ }^{3}$, avec qui il collabore au Feu, Gaillard va bouleverser l'esprit des Cahiers $d u$ Sud. Jean Ballard, dans une interview accordée à René Kochmann le 6 avril 1968 au siège de la revue, lui donne d'ailleurs une place capitale :

Mais en 1925, est survenu quelque chose de nouveau, est survenu un jeune homme, [...] un poète, qui était un homme passionné, une nature de feu, c'est Gaillard ! Et celui-là [...] a changé l'atmosphère des Cahiers du Sud. [...] Et Gaillard a [...], pour ainsi dire, canalisé vers les Cahiers presque tous ceux qui avaient un nom dans l'école surréaliste : Eluard, Michaux, Péret, Supervielle, Soupault, Vitrac, Artaud, et j'en oublie beaucoup encore.

Il est évident que l'atmosphère, le climat des Cahiers du Sud a complètement changé à cette époque. [...] Et c'est alors, depuis cette époque, que les Cahiers $d u$ Sud sont devenus une revue importante. De Paris nous vinrent des collaborations remarquables et il faut dire qu'à ce moment-là la revue des Cahiers $d u$ Sud ne fut plus une revue comme elle avait prétendue l'être, une revue marseillaise, une revue de province, elle devint une revue nationale, d'importance nationale. Bientôt nous prîmes rang parmi les premières. La revue européenne avait disparu, il n'y avait plus que La Nouvelle Revue française et la revue Europe. Eh bien je crois qu'avec ces deux-là, parmi les revues d'avant-garde, je veux dire celles qui prétendaient être le reflet de la nouvelle génération littéraire, nous formions le trio...

\footnotetext{
${ }^{1}$ André Gaillard lui-même les reniera.

${ }^{2}$ André Gaillard rencontre Philippe Chabaneix au lycée de La Rochelle et ils se lient d'amitié. Celui-ci est sans doute le poète le plus talentueux du groupe fantaisiste qui essaie alors de s'éloigner du symbolisme.

3 « Je me souviendrai toujours de ce dimanche d'octobre 1924 où il m'adressa un mot que je conserve encore. Je le rencontrais alors pour la première fois et pour la première fois aussi je voyais un homme pour qui la poésie était la seule raison de vivre. [...] André me délivra de l'alexandrinisme mais ce n'est pas le diminuer que de dire que lui aussi l'avait connu. [...] Octobre 1924, ou en étiez-vous au quai du Canal ? Depuis quatre ans dans Le Feu il parlait d'Aragon, de Breton, disait sa foi en la renaissance de l'esprit et vous n'en saviez rien. Une soirée aussi émouvante je ne crois plus en vivre d'autres. [...]. Son intelligence lucide éclairait tout, j'étais encore dans les ténèbres et j'ai compris qu'André me sauverait. Je te l'ai envoyé six mois plus tard et j'ai même eu beaucoup de peine à le décider. C'est par amitié pour moi qu'il a pris la résolution de te rencontrer. Tu sais combien il était difficile de le persuader mais tu as eu la chance de lui plaire et il a rêvé dès cette date de t'aider et de faire des Cahiers une puissance au service de l'esprit... Il régnait aux Cahiers une atmosphère conventionnelle et guindée qui me faisait regretter ce qu'il pouvait y avoir de superficiel chez toi au temps de Fortunio. », Léon-Gabriel Gros, [Lettre à Jean Ballard], 8 novembre 1930.
} 
Sous l'influence de Gaillard, les années 1925-1926 sont donc celles du changement : il est d'abord à l'origine d'un rajeunissement des sommaires et transforme l'ancienne revue mussettiste en une vraie revue d'avant-garde, et ce tant sur le plan poétique, que sur ceux de la critique et de la direction. Entre 1925 et 1928, il publie quarante et un poèmes en neuf numéros et soixante-quatre comptes rendus. Sa première apparition au sommaire de la revue marseillaise date du mois d'avril 1925 : il y publie le poème « Air de Bal », encore fortement lié au courant fantaisiste. La publication suivante, trois de ses poèmes, extraits du recueil $L a$ Terre n'est à personne, rassemblés sous le titre "Apocalypses », marque l'évolution du style de Gaillard mais aussi l'ouverture de la revue à l'avant-garde surréaliste. Ses poèmes ultérieurs, qu'il s'agisse des «Plaisirs de la vie brève » (dans le numéro d'octobre 1925) ou d'autres poèmes de La Terre n'est à personne (en décembre 1926) par exemple, conserveront leur caractère surréaliste. Les sommaires, durant cette période, sont impressionnants par les noms qui se côtoient: Michel Leiris donne sept textes, Roger Vitrac et Robert Desnos en donnent cinq, Antonin Artaud et Roger Ribemont-Dessaignes trois, tandis que René Crevel, Paul Eluard et Philippe Soupault sont chacun publiés à deux reprises.

Jean Ballard a alors assez d'intelligence et de liberté pour faire de Gaillard son homme de confiance et lui laisser un certain nombre de responsabilités. Jamais il ne s'oppose à cette nouvelle orientation, conscient de l'enrichissement qu'elle représente pour les Cahiers. Son ardeur et son opiniâtreté sont parfaitement complétées par le charisme de Gaillard. A ce propos, Georgette Camille, amie de Gaillard et correspondante des Cahiers à Paris, raconte : «A cette époque-là, Gaillard dirigeait réellement les Cahiers du Sud. Je me souviens, tout le monde passait par les Cahiers du Sud et allait voir Gaillard. Ballard était là, il était le directeur, mais il ne s'occupait pas tellement de nous. Il s'occupait beaucoup de la publicité. Il touchait de petites allocations de l'Etat. Il se débrouillait très bien. C'était un homme d'affaires » (52-3).

André Gaillard prend à cœur ce rôle de découvreur et de passeur que Ballard lui laisse avec une confiance étonnante. Afin de l'honorer, il se lance dans une exploration minutieuse du champ poétique, lisant les petites revues et écrivant à nombre de jeunes poètes, comme le raconte André Rolland de Renéville :

Je n'ai jamais connu André Gaillard, mais je crois pouvoir dire que je l'ai connu. Lorsque j'habitais Tours, ma ville natale, et que seul je m'efforçais d'approfondir cette voie de connaissances qu'on nomme la poésie, je recevais des lettres d'André Gaillard. Elles éclairaient ma solitude mentale. L'une d'elles contint l'offre de publier dans les Cahiers $d u$ Sud les premières pages d'un livre que j'écrivais alors sur Rimbaud. Et c'est ainsi que pour la première fois j'entrai par l'intermédiaire d'André en communication avec les amis inconnus que contient virtuellement le public. De Marseille où il donnait tous ses soins, et composait leur âme, au Cahiers $d u$ Sud, André Gaillard découvrait ainsi dans toutes les villes de province le jeune homme épris de poésie que chacune d'elles supporte (le mot n'est pas exagéré) et auquel ses signes d'intelligence apportaient un inaltérable réconfort. (850)

On le voit, un mot d'André Gaillard est, à l'époque, un «sauf-conduit pour le territoire poétique » (Fabre 21). Mais si Gaillard se lie d'amitié avec les jeunes poètes du Grand Jeu et qu'il leur prodigue des encouragements (avec André Rolland de Renéville mais aussi avec Pierre Minet, par exemple, publié pour la première fois dans le numéro des Cahiers de juin 1927), il garde néanmoins un regard critique sur leur travail. Le mois suivant le premier numéro de la revue, intitulée elle aussi Le Grand Jeu, à l'été 1928, il publie dans les Cahiers un commentaire à ce propos, considérant leur parution «nettement insuffisante quant aux textes poétiques » mais «par contre remarquable dans la partie critique », signalant particulièrement les essais de Maurice Henry, Roger Gilbert-Lecomte et René Daumal. Dans le deuxième numéro du Grand Jeu, Daumal et Gilbert-Lecomte évoquent cet encouragement 
extérieur dans la "Critique des critiques », affirmant qu' "André Gaillard avec une franche sympathie qui le met nettement de ce côté de la barricade, est en outre, une des rares personnes qui [leur] aient fait un reproche capable de [les] toucher et méritant d'être discuté » (Paire 25-6).

Comme le montre cette anecdote, André Gaillard avait une double casquette : ces critiques et comptes rendus qui réservent un accueil favorable à l'avant-garde, donnent aussi une nouvelle impulsion aux Cahiers. Il ne mentionne qu'une fois, durant ces années, les grands surréalistes comme Breton, Desnos ou Aragon. Yves Bridel, dans Miroirs du Surréalisme, note qu'il est le seul, en province, à parler de l'œuvre de René Char, et l'un des seuls à rendre compte des ouvrages de Leiris, Desnos, Vitrac ou encore Péret. La particularité des chroniques de Gaillard est le style dans lequel elles sont rédigées : bien souvent, elles ne prennent pas la forme d'une critique rationnelle et argumentée mais font allusion à l'ouvrage dans un style lyrique, presque poétique, qui évoque les émotions et les sentiments de l'auteur à sa lecture. A titre d'exemple, prenons le compte rendu de Gaillard à propos d'En joue de Philippe Soupault qui commence ainsi : «Le mouvement perpétuel des grandes roues de l'évasion n'est plus qu'un délire d'écureuils en cage et sur lui-même éternellement retournant » (71). A la suite de Gaillard, d'autres collaborateurs vont également parler de l'avant-garde : d'abord Georges Bourguet, qui signe le premier article de fond consacré au surréalisme, mais aussi Léon-Gabriel Gros et, à partir de 1928, Joë Bousquet ${ }^{4}$. Ces deux derniers, en particulier Bousquet, vont souvent adopter ce style, qui peut convenir lorsqu'on apprécie une œuvre, mais qui est plus critiquable quand il s'agit de l'attaquer. Si certains voient dans ces critiques proches du poème en prose une manière de mieux toucher la sensibilité du lecteur, d'autres les considèrent comme le refus de prendre le recul nécessaire à une saine critique.

Ces nouvelles intonations choquent le lectorat plutôt bourgeois de l'ancien Fortunio qui se désabonne alors en masse si l'on en croit Ballard: "Leur ton effraya vite les lecteurs traditionnels, le comble fut atteint avec la publication de Dormir, dormir dans les pierres de Benjamin Péret. [...] Les désabonnements pleuvaient. Nos amis du début nous croyaient fous et nous abordaient en se touchant le front »(Fabre 19). Ces réactions négatives l'amènent à préciser l'orientation générale de la revue, qui est ouverte à tout mouvement sincère et qui faisant parfois violence au public - quand l'esprit de révolte ou de fantaisie l'exige - ne s'astrei[nt] pourtant à aucun catéchisme d'école, choisi[t] librement ce qui plaît en chacune et donn[e] dans [son] sommaire une vision panoramique de la littérature. Il s'en suit que la plus grande liberté règne aux Cahiers $d u$ Sud, que les tendances les plus diverses y évoluent à l'aise, que les extrêmes s'y coudoient sans s'effaroucher ni s'exclure. (Ballard, 1927, 78)

Autrement dit, Ballard affirme une nouvelle fois l'indépendance d'une revue pour laquelle seules la qualité et la nouveauté comptent. Comme on le voit, Gaillard marque les Cahiers du Sud tant par son style que par « un ton nouveau, une exigence, une recherche, une aventure nouvelles [qui] s'installent grâce à [lui], et à l'exemple de ses textes, mais tout autant grâce à sa présence et à l'action de sa personne » (Bridel, 1987, 261).

Jusqu'alors, le rôle de rédacteur en chef était tenu par Gabriel d'Aubarède, ami de Marcel Pagnol, qui essayait d'attirer la revue vers le gidisme. Entre 1923 et 1925, il va régulièrement à Paris et s'y installe progressivement. Il commence alors à écrire pour la $N R F$ qu'il lie à son avenir littéraire. Mais lorsqu'André Gaillard arrive aux Cahiers, il provoque une véritable scission. Les débats houleux qui agitent l'équipe marseillaise n'ont pas laissé de traces parce qu'ils ont lieu lors des conversations journalières avec les collaborateurs. En revanche, nous possédons des lettres de Gabriel d'Aubarède puisqu'il demeure à Paris. Adhérant aux

\footnotetext{
${ }^{4}$ Joë Bousquet est déjà proche du courant surréaliste : ami avec Paul Eluard, il cosigne des tracts des 1925. C'est bien André Gaillard qui l'attire aux Cahiers du Sud.
} 
opinions plutôt défavorables de Gide et de la $N R F$ sur le surréalisme, il est en désaccord avec le tournant pris par la revue ${ }^{5}$ et démissionne le 18 décembre 1928 :

$\mathrm{J}^{\prime}$ ai lu de près le dernier numéro ${ }^{6}$, que je ne connaissais pas encore tout entier, et j'ai été désolé d'y trouver toujours de plus en plus dessiné un esprit combatif qui me semble déplorable. À côté d'une partie anthologique excellente [...], des articles ridicules, il n'y a pas d'autre mot : les dithyrambes de Neveux sur Desnos («l'éternité de Robert Desnos !»), les propos de Joë Bousquet sur Benjamin Constant ; les tirades de René Daumal sur Breton [...]. Beaucoup de personnes [...] me demandent les raisons d'une pareille attitude critique, et que puis-je répondre, étant le premier à la juger insoutenable ? [...]

$\mathrm{Tu}$ vas me trouver sévère. Je t'assure que je le suis moins que beaucoup. Je m'accuse d'ailleurs moi-même, qui pour avoir voulu faire preuve de libéralisme, ai montré en réalité de la faiblesse. Aujourd'hui il me faut te le dire, je ne me sens pas le courage d'endiguer un courant que je n'ai favorisé, tu le sais, qu'à contrecœur, et non d'ailleurs, sans m'être à plusieurs reprises élevé contre lui. [...] Du point de vue - il est vrai bien secondaire ici - de mon œuvre propre, et de ma carrière, je ne le pourrais sans créer autour de moi un malentendu compliqué, et dans mon esprit un trouble dont mon travail personnel souffrirait.

[...] Je reste et resterai quoi qu'il arrive l'ami des Cahiers, mais je ne vois vraiment pas le moyen [...] d'en assumer plus longtemps la rédaction.

Du reste, tu sais que certaines des qualités qui sont nécessaires à un tel poste me manquent absolument. [...] L'activité, la fermeté, le pouvoir d'être un animateur, tous ces dons il me semble que Gaillard les possède bien davantage. Et il me semblerait tout indiqué qu'il me remplace.

Même si Ballard choisit de ne pas donner la place de rédacteur en chef à Gaillard, qui restera toujours un homme de l'ombre, mais à Gabriel Bertin, la recommandation de d'Aubarède est le signe de l'influence de Gaillard qu'il perçoit comme le plus capable et le plus représentatif de l'évolution de la revue.

Un incident vient précipiter le départ de Gabriel d'Aubarède : dans le numéro de décembre 1928 consacré à Marseille, le nom de Pierre Audibert est caviardé suite à un malentendu avec l'imprimerie. Pour éviter à Audibert, mêlé à un scandale, de se ridiculiser encore plus, Ballard demande à réimprimer l'article sans signature. Malheureusement, l'imprimeur se contente de gratter le nom. Ce caviardage provoque la colère de Gabriel d'Aubarède qui, mis en cause par Candide, ordonne à Ballard de retirer son nom de la revue. Ce même événement pousse Bousquet à quitter les Cahiers, par amitié pour Eluard qui lui écrit le 13 février 1929: «Si cette revue dure, je considérerai [...] tous ses collaborateurs comme mes ennemis personnels » (Eluard 72). Au même moment, le nom de Gaillard disparaît aussi des sommaires (il ne participe à aucun numéro de 1929). Il est difficile de croire à un simple hasard. Est-ce aussi par amitié pour Eluard ? On sait seulement par Léon-Gabriel Gros qu'il a connu une grave « crise » durant l'hiver 1928-1929 ${ }^{7}$. On ignore à quoi correspond cette crise :

\footnotetext{
${ }^{5}$ Il écrit déjà le 15 mai 1928 : «Lu l'article sur les Cahiers dans la N.R.F. Je t'avoue qu'il ne me cause pas que du plaisir, car il me semble donner le ton de ce qu'on pense en général de notre orientation littéraire, et elle ne correspondrait nullement ni à mes goûts ni à mes idées. [...] Il m'est toutefois pénible de lire qu'une revue dont je suis, en principe, le rédacteur en chef, passe pour avoir une doctrine, et que cette doctrine serait une sorte de post-surréalisme. On dirait que j'ai poussé les Cahiers $d u$ Sud dans une voie qui est celle pour laquelle je ressens le moins d'attrait. D'où un malentendu qui risque de s'établir sur mon propre état d'esprit en matière de littérature. De deux choses l'une, ou je vais passer pour avoir des sympathies envers ce mouvement (d'ailleurs mort) et je ne veux assumer ni cet aveuglement ni ce ridicule ; ou bien et plus justement je vais passer pour un rédacteur faible et tout passif, une sorte de trieur de manuscrits indulgent et qu'on pousse où l'on veut, et cette opinion est désobligeante. », Gabriel d'Aubarède, [Lettre à Jean Ballard], 15 mai 1928.

${ }^{6}$ Il s'agit du numéro 106 des Cahiers du Sud de novembre 1928.

${ }^{7}$ «Seul André a pu me décider à travailler encore et ma grande consolation restera toujours de l'avoir aidé au cours de cette crise de l'hiver dernier où la comédie même qu'il jouait m'a persuadé de la confiance qu'il me portait. », Léon-Gabriel Gros, [Lettre à Jean Ballard], 8 février 1930.
} 
est-elle due à la démission de d'Aubarède, au caviardage, à des problèmes de santé ${ }^{8}$ ? Est-ce une remise en question personnelle ? Rien ne nous permet de répondre à cette question et la mort brutale d'André Gaillard le 16 décembre 1929 rajoute encore des interrogations. Ce décès tragique - il meurt, officiellement, d'une congestion cérébrale - donne un coup de frein à la revue. Georgette Camille rapporte cette anecdote : «A la mort de Gaillard, j'ai dit à Ballard : "Il faut arrêter les Cahiers $d u$ Sud, parce qu'on ne peut plus continuer maintenant qu'André Gaillard n'est plus là." Il m'a dit : "Mais non, mais non, je ne peux pas arrêter." C'était sa vie [...]. Alors il a continué avec les Cahiers du Sud après la mort de Gaillard » (53).

Les mois qui suivent attestent nettement de la perte de vitesse des Cahiers qui ont perdu leur plus grand passeur. La plupart des auteurs du Grand Jeu, par exemple, qui ont trouvé aux Cahiers une rade salutaire, s'éloignent de la revue comme le rappelle Ballard à René Daumal le 30 avril 1932 : «Depuis la mort de Gaillard les principaux membres du « Grand Jeu » n'ont pas suffisamment collaboré aux Cahiers [...]. Hormis Rolland de Renéville, et très accidentellement d'ailleurs, ni vous, ni Lecomte, ni Vaillant, ni Delons (tout au moins jusqu'à présent) n'avez figuré aux sommaires ». De même, lorsque Léon Pierre-Quint, à qui Ballard a transmis le flambeau, cherche à amener de nouvelles collaborations à la revue, il se heurte à un certain nombre de réticences, comme il le lui écrit le 18 octobre 1930 :

Je ne peux pas vous cacher que je rencontre une assez grande hostilité quand je demande des collaborations aux Cahiers $d u$ Sud. Les écrivains qui vous connaissent personnellement, eux, n'hésitent pas à collaborer ; ceux qui ne vous connaissent pas, n'ayant pas d'opinion sur vous, regrettent Gaillard - évidemment, et sont plein de préjugés au sens étymologique du mot.

Il est clair que les Cahiers se remettent difficilement de cette perte, mais Ballard fait le choix de continuer et l'histoire lui donne raison. Pour autant, l'équipe des Cahiers ne cesse de reconnaître l'importance des apports de ce médiateur qui a mis de côté sa propre carrière pour révéler le talent d'autres poètes. Durant les années suivantes, toute décision est soumise au jugement imaginaire de l'absent. Pendant dix ans, chaque année, dans le numéro de décembre, un article rend hommage à André Gaillard.

Avec le recul, nous sommes les témoins de l'incroyable flair littéraire d'André Gaillard, de sa clairvoyance qui révèle les étonnantes capacités d'anticipation de celui qui a su, par exemple, reconnaître le talent de René Daumal ou encore de Joë Bousquet. Il est donc le premier compagnon de Ballard qui lui permet de renforcer et de renouveler les découvertes littéraires : c'est un (ré)novateur. Ce passeur d'idées et de textes - passeur furtif tant par la brièveté de sa collaboration aux Cahiers que par une position volontairement en retrait, est parvenu à transformer une commune petite revue de province en une revue de renommée internationale; mais surtout, il en a fait l'un des meilleurs laboratoires de la littérature contemporaine dans cette période d'entre-deux-guerres. Ballard, saisi par cette ferveur, se laisse entraîner à l'opposé de ses propres goûts : en quelques années, la revue sort de sa Provence natale, trouvant sa place dans l'espace littéraire où les grandes revues parisiennes dont la NRF de Jean Paulhan ${ }^{9}$ - saluent sa venue. Bref, en quatre ans, les Cahiers du Sud entrent dans l'histoire littéraire.

\footnotetext{
${ }^{8}$ Dans une lettre adressée à Joë Bousquet en février 1929, Eluard affirme que Gaillard est « malade » et qu'il vient d'abandonner les Cahiers $d u$ Sud.

9 «Ils sont aujourd'hui une revue assurée, qui a sa doctrine littéraire, ses disciples, et qui a révélé, en 1927, plus de poètes et d'écrivains étrangers que n'ont fait deux à trois de nos grandes revues réunies. », Jean Guérin (pseudonyme de Jean Paulhan), NRF, 176, mai 1928.
} 


\section{Ouvrages cités}

Aubarède, Gabriel (d'). «A la dérive par Philippe Soupault ». Fortunio. 51, 15 mars 1924 : 320-1.

Ballard, Jean. «Explications ». Cahiers du Sud, 86, janvier 1927 : 77-80.

$1963: 25-8$.

Bridel, Yves. Miroirs du surréalisme. Paris : L'Age d'Homme, 1988.

—. «Les Cahiers du Sud et André Gaillard (1924-1929) ». Sud. 71-72, $1987: 261$.

Camille, Georgette. «Nous vivions dans un état de poésie totale », témoignage recueilli par Blandine Masson. La Revue des revues, revue internationale d'histoire et de bibliographie. 16, 1994 : 51-3.

Eluard, Paul. Lettres à Joë Bousquet. Paris : Les éditeurs français réunis, 1973.

Fabre, Daniel. Cahiers du Sud : la génération de 1930. Paris : Centre National des Lettres : Ent'Revues, 1987.

Gaillard, André. «En joue par Philippe Soupault ». Cahiers du Sud. 75, janvier 1926 : 71.

Paire, Alain. «Les Cahiers du Sud et le Grand Jeu (1937-1944) ». La Revue des revues, revue internationale d'histoire et de bibliographie. 14, 1992 : 25-36.

Rolland de Renéville, André. «Les poètes et la société ». Cahiers du Sud. 178, décembre $1935: 850$. 